\title{
AN EXAMPLE OF ANN MODELLING APPLICATION IN PATCH LOADING PROBLEMS OF STEEL STRUCTURES
}

\author{
Dr Biljana Šćepanović* \\ University of Montenegro, Faculty of Civil Engineering, Podgorica, Montenegro \\ Dr Miloš Knežević \\ University of Montenegro, Faculty of Civil engineering, Podgorica, Montenegro \\ Dr Duško Lučić \\ University of Montenegro, Faculty of Civil Engineering, Podgorica, Montenegro
}

Three different collapse modes are observed in experimentally tested eccentrically patch loaded steel l-girders: eccentric, centric and mixed collapse mode. The most important difference between collapse modes is in collapse load. Numerous mutually dependant and related parameters, as well as their combinations influence the behaviour, collapse mode and collapse load of eccentrically patch loaded steel l-girders. Dealing with such a big number of correlated influential parameters, makes determination of collapse mode and calculation of collapse load difficult tasks. One approach that was analysed and assessed as successful method is application of Artificial Neural Networks $(A N N)$.

Keywords: patch load, eccentricity, steel l-girder, collapse mode/load, ANN modelling, forecast model

\section{INTRODUCTION}

A series of experimental researches analysing patch loading problems was organised at the University of Montenegro, since 1998 [1-6]. Among the other issues, particular attention was paid to the problem of eccentric patch loading, having in mind that a certain eccentricity of load, regarding the web plane, is unavoidable in real structures. Fig. 1 shows tested girder with characteristic dimensions. Girder span, a, and web depth, hw, as well as flange width, bf, are same in all tested girders $\left(a=h_{w}=700 \mathrm{~mm}, b_{f}=150\right.$ $\mathrm{mm}$ ). Web thickness, tw, flange thickness, $t_{f}$, as well as load eccentricity, $e$, are variables $\left(t_{w}=3 \div\right.$ $10 \mathrm{~mm}, t_{f}=3 \div 15 \mathrm{~mm}, e=0 \div 30 \mathrm{~mm}$ ), making also variable influential parameters as $e / b_{f}, b_{f} / t_{f}$ $t_{f} / t_{w^{\prime}}, h_{w} / t_{w}$.

For the analysis of eccentric patch loading, 144 tests were done in the experiments from 1998 [1, 2], 2001 [3, 4] and 2007 [5, 6]. Girders are
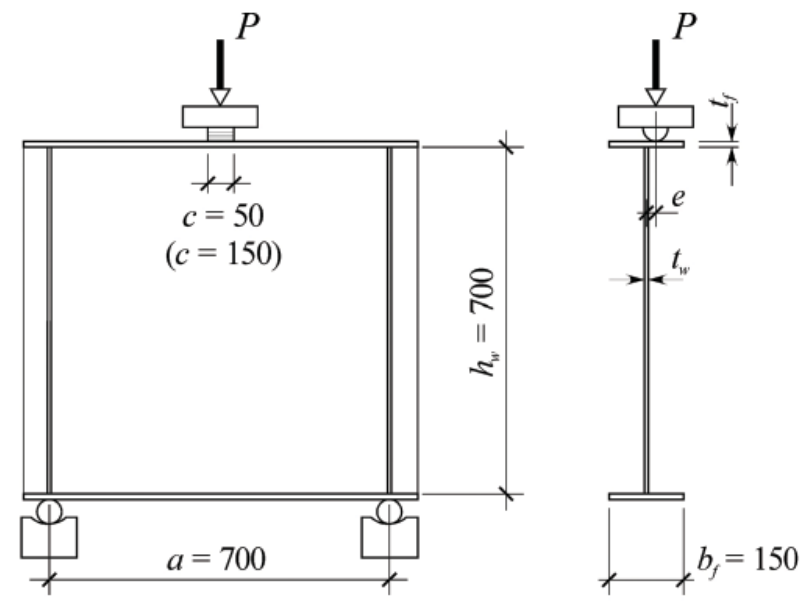

Figure 1. Experimentally tested I-girder under eccentric patch loading 
grouped in series, by the dimensions. Six girders of same geometry make one series where load eccentricity varies in range from $e=0$ to $\mathrm{e}=b_{f} / 6=25 \mathrm{~mm}$. A few girders were also tested with eccentricity $e=30 \mathrm{~mm}$. Most girders were loaded over the length $c=50 \mathrm{~mm}$, while several samples had load length $c=150 \mathrm{~mm}$.

It was shown that the collapse mode of most (but not all!) girders subjected to eccentric patch loading was quite different from the collapse mode of centrically loaded girders. Three different collapse modes are observed in experimentally tested eccentrically patch loaded steel I-girders: eccentric [1-6], centric [1-6] and mixed $[5,6]$ collapse mode. Mixed collapse mode, having characte 7 ristics of both, centric and eccentric collapse modes, may appear in two variants: as centric-mixed or as eccentric-mixed collapse mode, depending on dominant collapse mode characteristics [6].

Concerning engineering practice, the most important difference between collapse modes is in ultimate load. The reduction in ultimate load with an increase in load eccentricity is obvious in girders with eccentric collapse mode. For a certain girder geometry, even the smallest load eccentricity $\left(e=5 \mathrm{~mm}\right.$, i.e. $\left.e / b_{f}=1 / 30\right)$ reduced ultimate load over $40 \%$. In case of centric collapse mode in eccentrically loaded girders, ultimate load does not change significantly with an increase in load eccentricity. Even for highest load eccentricity $\left(e=25 \mathrm{~mm}\right.$, i.e. $\left.e / b_{f}=1 / 6\right)$, girders of certain geometry behaved as if there is no eccentricity.

Hence, in order to estimate ultimate load, the first step is to determine collapse mode of eccentrically loaded I-girder. Influential parameters are numerous, mutually dependant and related: girder geometry (all dimensions of girder and their ratios), load eccentricity and its relations with girder dimensions, as well as load application manner. Dealing with such a big number of correlated influential parameters, makes determination of collapse mode and calculation of collapse load difficult tasks. One approach that was analysed is application of Artificial Neural Networks (ANN).

ANN modelling method is based on the analogy with the human nervous system [6, 7]. Artificial neuron imitates biological neuron. Artificial Neural Network, consisted of artificial neurons, is computational simulation of human neural network, consisted of biological neurons. Humans use their mind to make conclusions and decisions in certain situations based on the previous (similar) experience. ANN does not have human mind and experience that should be used to process input data and make appropriate conclusions/decisions, i.e. output. In ANN modelling method human mind is replaced by mathematical functions (as much as such replacement is possible) and human experience is replaced by existing data base which is used for ANN training. By training ANN on some data base, forecast models are created in order to estimate output parameter(s) for certain set of input parameters that is not present in the data base, but that is in the range of data base.

\section{ANN MODELLING OF EXPERIMENTALLY TESTED GIRDERS}

Although the problems of collapse mode determination and collapse load calculation are mutually connected, collapse mode qualifying the level of collapse load, for the beginning these two issues are considered independently from each other. At first, Artificial Neural Network modelling was implemented in estimation of collapse load, without considering collapse mode. Latter on, the same networks, that gave good results in estimation of collapse load, were slightly modified, adapted and used to forecast collapse mode.

\section{FORECAST MODELS FOR COLLAPSE LOAD}

The basic idea was to estimate the collapse load, $\mathrm{Pu}$, as the only output parameter, depending on numerous input parameters (material characteristics, girder geometry and load eccentricity), as well as to asses applicability of ANN modelling method for collapse load determination in engineering practice.

Several types of forecast models were made using experimental data from 1998, 2001 and 2007: with dimensional (e.g. $e, t_{f}, t_{w}$ ) and dimensionless (e.g. e/b $b_{f}$ e/t $, e / t_{w}, b_{f} / t_{f}, t_{f} / t_{w^{\prime}} h_{w} / t_{w}$ ) geometry inputs. Several types of network architecture were constructed: with one or two hidden levels of neurons; with different number of neurons (1 to 20) in each level, depending on inputs number and number of training data. Later on, comparison of different forecast models was done in order to evaluate which models provide the best forecast of collapse load. Afterwards, these best 
models were used for assessing applicability of ANN modelling method in engineering practice, as a tool for determination of collapse load in eccentrically patch loaded steel I-girders.

Only one computer software $[6,7]$ was used for training of all created Artificial Neural Networks, so that the following characteristics were the same for all networks, i.e. for all ANN forecast models:

- The training of feed-forward neural networks was done by means of improved back-propagation algorithm that assumes iterative training process.

- The number of training cycles in one iteration was 500 . Iterative process of network training, i.e. model fitting was stopped when error of validation data set showed intention to grow after lessening trend. The error of training data set constantly decreased.

ANN models were made separately for girders with different load lengths ( $c=50$ or $150 \mathrm{~mm}$ ). Herein only load length of $c=50 \mathrm{~mm}$ and models with five dimensional inputs $\left(e, t_{w}, t_{f,} \sigma_{0.2, w}-\right.$ web yielding stress, $\sigma_{0.2, \mathrm{f}}-$ flange yielding stress) and one output $(P u)$ will be considered. The com- plete experimental data set for girders with load length $c=50 \mathrm{~mm}$ consists of 120 tested girders. 19 testes were exempted from the network training process and used as a comparison data set, i.e. as data for the evaluation of forecast models. The rest of 101 tests were divided in training data set (71 tests) and validation data set (30 tests).

The best evaluated models show high level of match with experimental data and prove to be acceptable for engineering practice. Particularly good results are obtained from network with two hidden levels, each with ten neurons ("c50 - load - 2 - 10") [6]. Examples of collapse load forecast models of this network are presented in Figure 2.

Figure 2. illustrates estimation of collapse load and its relation with the load eccentricity $\mathrm{Pu}$,ann(e) for tw $=5 \mathrm{~mm}$ and different values of $\mathrm{t}_{\mathrm{f}}=5 \div 15 \mathrm{~mm}$, all at fixed $\mathrm{c}=50 \mathrm{~mm}, \sigma_{0.2, \mathrm{w}}=28$ $\mathrm{kN} / \mathrm{cm} 2$ and $\sigma_{0.2, \mathrm{f}}=28 \mathrm{kN} / \mathrm{cm} 2$. Some of these values of $t f$ have been tested experimentally $\left(t_{f}=\right.$ $6,8,10$ and $12 \mathrm{~mm}$ ). However, ANN models fill in the gaps for values that were not present in the experiment and also widen domain of $\mathrm{tf}$ values. Appropriate graphical presentation of $P u$,ann(e) for fixed value of $t_{f}$ and different values of $t w$

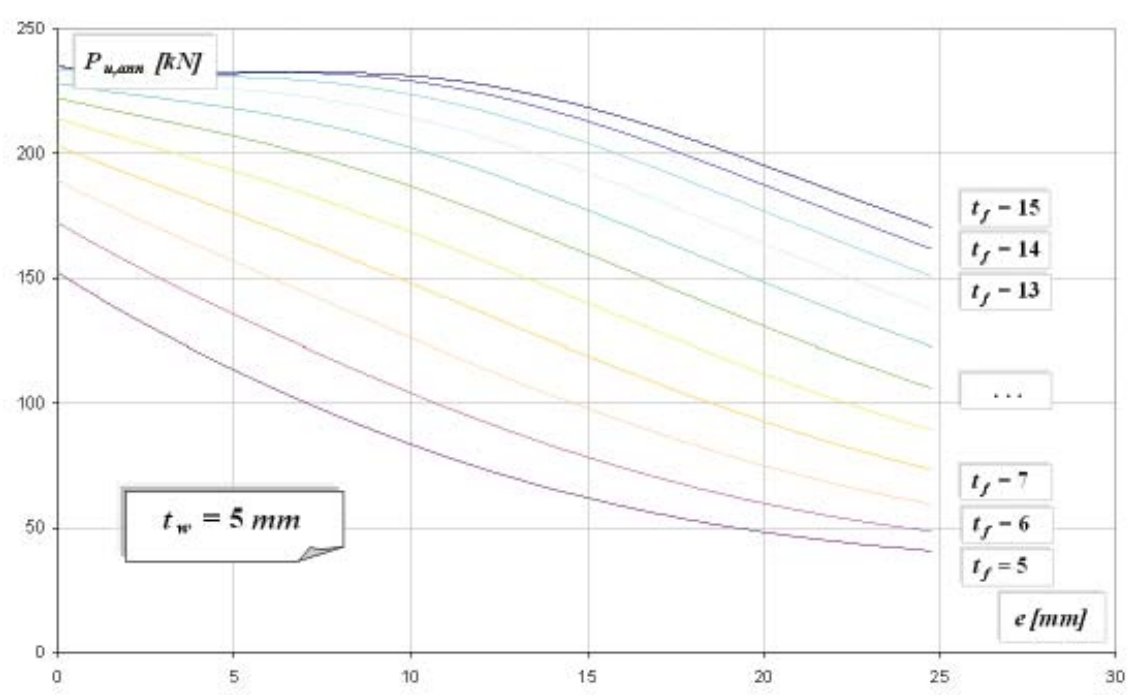

Figure 2. Estimation of collapse load Pu, ann (e) for $t_{w}=5 \mathrm{~mm}$ and $t_{f}=5 \div 15 \mathrm{~mm}$, at $c=50 \mathrm{~mm}, \sigma_{0.2, \mathrm{w}}=28 \mathrm{kN} / \mathrm{cm} 2$ and $\sigma_{0.2, f}=28 \mathrm{kN} / \mathrm{cm} 2$,

by means of Artificial Neural Network "c 50 - load - 2 - 10"

might also be created, assuming fixed values of c, $\sigma_{0.2, w}$ and $\sigma_{0.2, \mathrm{f}}$. Similar estimations of collapse load and its relations with the web thickness or dimensionless parameter $t_{f} / t_{w}$, i.e. $P u$,ann $\left(t_{w}\right)$ or $P u$, ann ( $t f / t w)$, might be made, as well. Such diagrams proved to be interesting, leading to various and important conclusions.

\section{FORECAST MODELS FOR COLLAPSE MODE}

Initial intention was to train again all networks used for collapse load estimation, but with the new output. However, some networks, showing obvious and persistent non-adequate behaviour 
from the early stages of iterative training, were abandoned during the training process. Instead of them, some new networks were constructed. Still some networks created for collapse load estimation, after appropriate adaptation, were successfully used for the collapse mode forecast. Input parameters and network architecture were kept the same. Only output parameter was changed. Collapse mode was introduced instead of collapse load and the same iterative network training process was done again, with the new output. Comparison, training and validation data sets were the same as for collapse load models.

In order to use available software [6, 7] for network training, it was necessary to transform collapse mode, being alpha-numerical, descriptive parameter, in the form of numerical parameter. Each type of collapse mode was assigned a numerical value from the interval $[-1,1]$ : centric col- lapse mode $=1$; centric-mixed $=0.3$; eccentricmixed $=-0.3$; eccentric $=-1$.

Among created networks, the best results are obtained from the following two [6]: network with one hidden level, with 6 neurons ("c50 - mode $-1-6$ "), and network with two hidden levels, each with 19 neurons ("c50 - mode - 2-19").

Examples of collapse mode forecast models of these networks are presented in Fig. 3 and Fig. 4. Four characteristic girder geometry types, i.e. four experimental series having representative sample in comparison data set were chosen for this presentation and comparison of ANN modelling of collapse mode with experimental results. Graphical presentation of results is chosen as the most suitable, having in mind character of output parameter - collapse mode. In this manner, visual evaluation of forecast models and assessment of ANN modelling application is enabled.
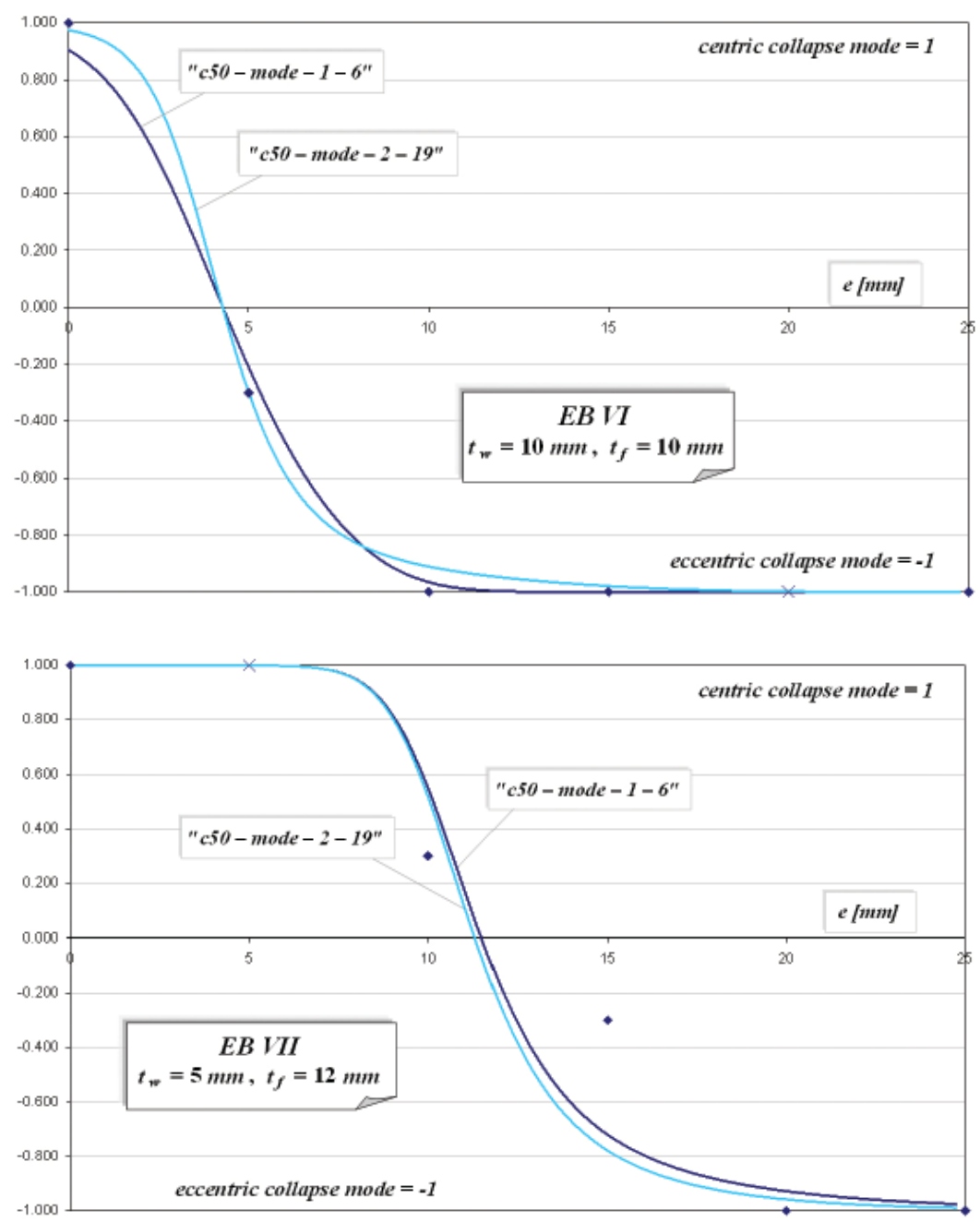

Fig. 3. Examples of collapse mode forecast models in experimental series EB VI and EB VII, for networks "c50 - mode - 1-6" and "c50-mode - 2-19" 
In Figure 3 and Figure 4, lines present ANN forecast models for collapse mode, while dots present experimental results. X-dot $(x)$ is sample from comparison data set. Square-dots $(\bullet)$ are samples of same geometry as that one, but with different load eccentricity. Each of four diagrams presents one complete experimental series, i.e. girders with the same dimensions, differing only in load eccentricity: five girders presented with square-dots $(\bullet)+$ one girder presented with $X$-dot $(x)=6$ girders of one experimental series. Load length in all presented series was $\mathrm{c}=50 \mathrm{~mm}$.

Generally speaking, presented ANN forecast models match experimental data well, what makes them applicable in practice. Noticeable discrepancy is observed only in case of centric collapse at high eccentricity of load, Fig. 4. It happens in girders with high ratio tf /tw. Only collapse mode for highest eccentricity $(e=25$ $\mathrm{mm}$ ) is not well forecasted, while ANN models provide good forecast of collapse mode for other eccentricities (e $\leq 20 \mathrm{~mm}$ ) even in these girders. In case of series with "more expectable", "more common" behaviour - centric behaviour of eccentrically loaded girders only up to the certain level of load eccentricity - ANN forecast modes have excellent match with experimental data, as shown in Figure 3.

\section{CONCLUSION WITH REMARKS}

Presented example of ANN modelling proves possibility of successful and useful application of this method both, in engineering practice for the purpose of determination of collapse load and collapse mode in eccentrically patch loaded steel I-girders, as well as in scientific research, in order to better understand the character and level of influence of certain parameters on collapse load and collapse mode.
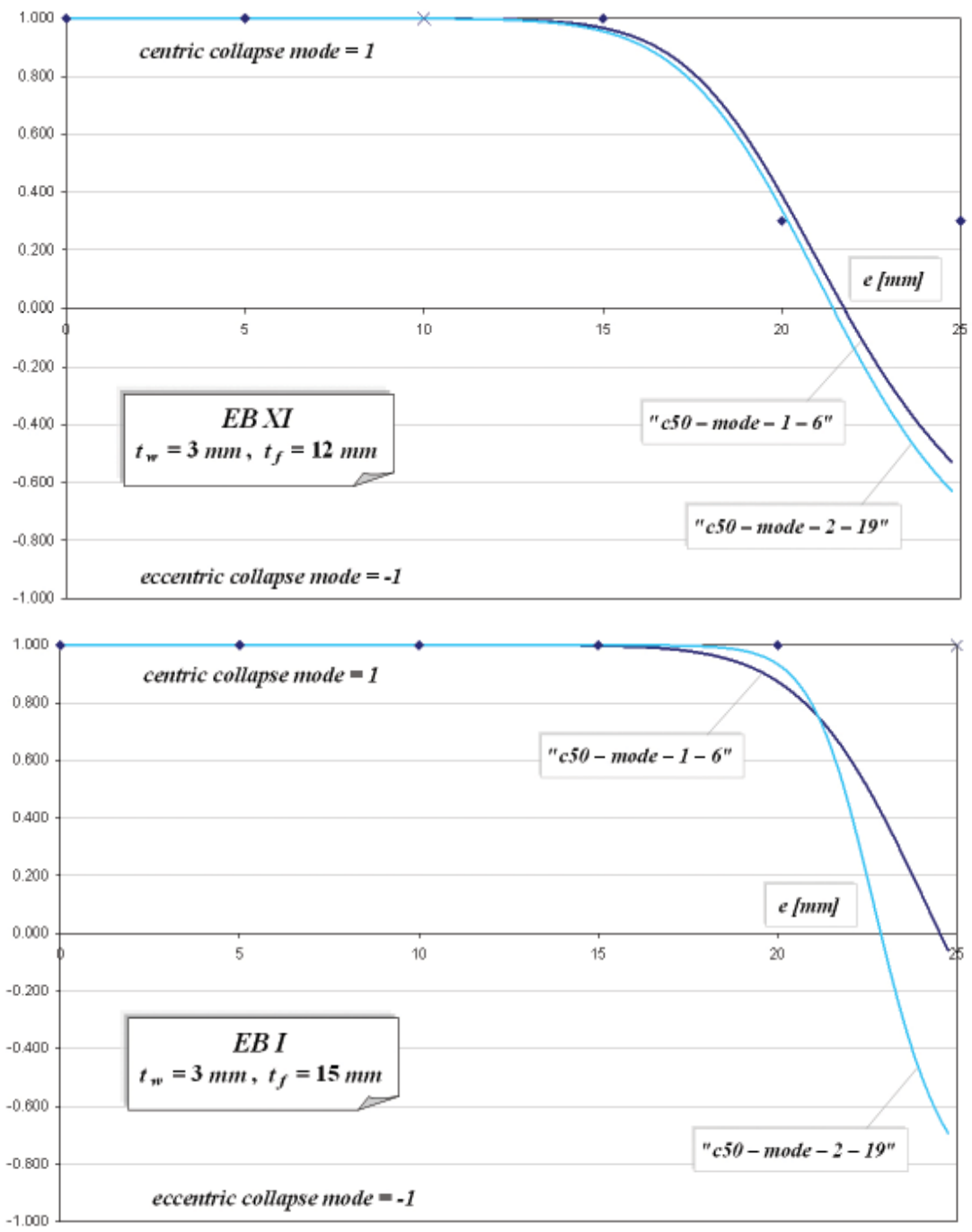

Figure 4. Examples of collapse mode forecast models in experimental series EB XI and EB I, for networks "c50-mode - 1-6" and "c50-mode - 2-19" 
Not only that ANN models might be used to forecast collapse load of particular girders. Graphical presentation of such models as in Fig. 2 might help in establishing relations between collapse load and certain influential parameters. It may also be useful in making conclusions about correlation and interaction among influential parameters.

Not only that ANN models might be used to forecast collapse mode of particular girders. They might also help in establishing general criteria for collapse mode identification in eccentrically patch loaded girders. One set of such criteria is formulated [6], based on existing experimental database. It means that proposed criteria are valid for the domain of experimental data. The question is what about girders with the dimensions out of experimental data domain. Graphical presentation of ANN models as in Fig. 3 and Fig. 4 might help answering this question. Diagrams in these Figures clearly imply separation of collapse mode types in girder of certain geometry, depending on load eccentricity. Zones of centric, mixed and eccentric collapse modes might be identified. By means of such diagrams, adequate ANN forecast models might provide new criteria for collapse mode identification, as well as their validation out of the experimental domain.

Only one example is presented here, aiming picturing successful application of ANN modelling method in subject problem solving. However, further work in this domain should be welcomed. Even available experimental database used for network training in this example might provide different and better results. Improvement is possible through: another choice of numerical values for collapse mode types; another choice of data for comparison, training and validation sets; another choice of Artificial Neural Network type, i.e. type of its architecture and training process; use of another computer software. Any future experimental or numerical (e.g. finite element method) modelling will provide extension of database, enabling creation of ANN models with better results.

Still, the fact that ANN modelling method belongs to the domain of artificial intelligence, should be kept in mind, since such methods should be strongly controlled by human intelligence in order to give results that comply with the reality.

\section{REFERENCES}

1) D. Lučić: "A contribution to the analysis of thin-walled girders", PhD thesis, Faculty of Civil Engineering, University of Belgrade, Belgrade, 1999.

2) D. Lučić: "Experimental Investigation on Igirders Under Eccentric Patch Loading", The 2nd European Conference on Steel Structures (EUROSTEEL 1999), Vol. 1, pp. 47-50, Prague, 1999.

3) D. Lučić, B. Šćepanović: "Experimental Investigation on Locally Pressed I-beams Subjected to Eccentric Patch Loading", The 3rd European Conference on Steel Structures (EUROSTEEL 2002), Vol. 1, pp. 473482, Coimbra, 2002; Journal of Construction Steel Research, Vol. 60, Nos. 3-5, pp. 525534, 2004.

4) B. Šćepanović: "I-girders under eccentric local loading - experimental-theoretical analysis", MSc thesis, Faculty of Civil Engineering, University of Belgrade, Belgrade, 2002.

5) B. Šćepanović, D. Lučić, S. Aleksić: "An Experimental Research Ekscentro 2007 - Experimental Testing of Eccentrically Patch Loaded Steel I-Girders", The 5th European Conference on Steel and Composite Structures (EUROSTEEL 2008), Vol. B, pp. 11491154, Graz, 2008.

6) B. Šćepanović: "Analysis of eccentrically locally loaded steel I-girders", PhD thesis, Faculty of Civil Engineering, University of Montenegro, Podgorica and Faculty of Civil Engineering, University of Granada, Granada, 2010.

7) M. Knežević: "Risk management during realisation of the civil engineering projects", $\mathrm{PhD}$ thesis, Faculty of Civil Engineering, University of Belgrade, Belgrade, 2004.

Paper sent to revision: 07.03.2012.

Paper ready for publication: 28.03.2012. 